\title{
A SYNTHETIC APPROACH TO SUBSTITUTED CYCLOPROPANES BASED ON THE COUPLING REACTION OF LITHIATED CHLOROALKYLOXAZOLINES WITH FISCHER CARBENE COMPLEXES
}

Saverio Florio, ${ }^{* a}$ Filippo M. Perna, ${ }^{a}$ Renzo Luisi, ${ }^{a}$ José Barluenga,${ }^{* b}$ Félix Rodríguez, ${ }^{b}$ and Francisco J. Fañanás,

${ }^{\mathrm{a}}$ Dipartimento Farmaco-Chimico, University of Bari, Via E. Orabona 4, I-70126-Bari, Italy

C.N.R., Istituto di Chimica dei Composti Organometallici "ICCOM", Sezione di Bari,

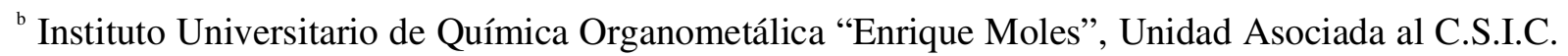
Julián Clavería, 8, Universidad de Oviedo, 33006 Oviedo, Spain 


\section{Experimental Section}

General. Tetrahydrofuran (THF) and diethyl ether $\left(\mathrm{Et}_{2} \mathrm{O}\right)$ were freshly distilled under a nitrogen atmosphere over sodium/benzophenone ketyl. All other chemicals were of commercial grade and used without further purification. Commercial solutions of $n$-BuLi (2.5 M solution in hexanes) were titrated by using $N$-pivaloyl-o-toluidine prior to use. ${ }^{1}$ For the ${ }^{1} \mathrm{H}$ and ${ }^{13} \mathrm{C}$ NMR spectra $(300,500$ $\mathrm{MHz} ;{ }^{13} \mathrm{C}$ NMR 75.4, $125 \mathrm{MHz}$ ), $\mathrm{CDCl}_{3}$ was used as the solvent. Carbon multiplicities were assigned by DEPT techniques. GC-MS spectrometry analyses were performed on a gas chromatograph HP 5890 II (dimethylsilicon capillary column, $30 \mathrm{~m}, 0.25 \mathrm{~mm}$ i.d.) equipped with a mass selective detector operating at $70 \mathrm{eV}$ (EI). Elemental analyses were carried out on a PerkinElmer 2400 and Carlo Erba 1108 microanalyzers. Melting points were uncorrected. TLC was performed on Merck silica gel plates with F-254 indicator; visualization was accomplished by UV light $(254 \mathrm{~nm})$ or by exposing to $I_{2}$ vapor. All reactions involving air-sensitive reagents were performed under nitrogen in oven-dried glassware using syringe-septum cap technique. The compounds 3a-e were prepared according to the reported procedure. ${ }^{2}$

Preparation of carbene complexes 4 and cyclopropanecarboxylates 5, 6, and 7. General Procedure.

To a solution of LDA, generated by reaction of $N, N$-diisopropylamine $(1.2 \mathrm{mmol})$ and BuLi (1.2 mmol, $0.48 \mathrm{~mL}$ of a $2.5 \mathrm{~N}$ solution in hexanes) in THF $(5 \mathrm{~mL})$ was added a solution of the corresponding oxazoline $1(1.0 \mathrm{mmol})$ in $\mathrm{THF}(2 \mathrm{~mL})$ at $-98^{\circ} \mathrm{C}$ under a nitrogen atmosphere. The mixture was stirred for $10 \mathrm{~min}$ and then a solution of the corresponding carbene complex $\mathbf{3}$ in THF $(5 \mathrm{~mL})$ was added dropewise. The reaction mixture was kept at $-98{ }^{\circ} \mathrm{C}$ for other $30 \mathrm{~min}$, then allowed to warm to room temperature and, once checked the consumption of $\mathbf{3 a}$, filtered through

\footnotetext{
${ }^{1}$ Suffert, J. J. Org. Chem. 1989, 54, 509.

${ }^{2}$ Aumann, R.; Heinen, H. Chem. Ber. 1987, 120, 537
} 
celite and silica gel. The solvent was removed under reduced pressure and the crude residue was purified by flash column chromatography (ethyl acetate/hexane: 1/9) to give the cyclopropylcarbene complexes 4 .

In order to obtain cyclopropanecarboxylate derivatives $\mathbf{5 , 6}$, and $\mathbf{7}$, the solution of the corresponding cyclopropylcarbene complex in hexane $(30 \mathrm{~mL})$ was subjected to the air oxidation under sun light. After $16 \mathrm{~h}$ the mixture was filtered off on celite, the solvent removed and the residue purified by column chromatography (AcOEt 1/ petroleum ether 9) to give the cyclopropane carboxylates $\mathbf{5}, \mathbf{6}$, and 7. Alternatively, the oxidation can be carried out with pyridine N-oxide in THF.

Pentacarbonyl-\{[(1R*,2R*,3S*)-2-(4,5-dihydro-4,4-dimethyl-2-oxazolyl)-2-methyl-3phenylcyclopropyl]methoxymethylene\}tungsten (4d): yellow solid, mp $43-44{ }^{\circ} \mathrm{C}, 80 \% .{ }^{1} \mathrm{H}$ NMR $\left(300 \mathrm{MHz}, \mathrm{C}_{6} \mathrm{D}_{6}\right) \delta 1.01(\mathrm{~s}, 3 \mathrm{H}), 1.11(\mathrm{~s}, 3 \mathrm{H}), 1.44(\mathrm{~s}, 3 \mathrm{H}), 3.20(\mathrm{~d}, J=6.2 \mathrm{~Hz}, 1 \mathrm{H}), 3.39$ and 3 $.49(2 \times \mathrm{d}, \mathrm{AB}$ system, $J=8.1 \mathrm{~Hz}, 2 \mathrm{H}), 3.92(\mathrm{~s}, 3 \mathrm{H}), 5.23(\mathrm{~d}, J=6.2 \mathrm{~Hz}, 1 \mathrm{H}), 7.04-7.24(\mathrm{~m}, 5 \mathrm{H})$; ${ }^{13} \mathrm{C}$ NMR $\left(75.4 \mathrm{MHz}, \mathrm{C}_{6} \mathrm{D}_{6}\right) \delta 16.5\left(\mathrm{CH}_{3}\right), 28.4\left(\mathrm{CH}_{3}\right), 28.5\left(\mathrm{CH}_{3}\right), 40.8(C \mathrm{H}), 41.2\left(C_{q}\right), 60.1$ $\left(\mathrm{CH}_{3}\right), 68.1(\mathrm{CH}), 69.7\left(C_{q}\right), 79.9\left(\mathrm{CH}_{2}\right), 127.4(\mathrm{CH}), 128.1(2 \times \mathrm{CH}), 128.3(2 \times C \mathrm{H}), 138.1\left(C_{q}\right)$, $163.7(C=\mathrm{N}), 198.4(4 \times C=\mathrm{O}), 204.8(C=\mathrm{O}), 327.5(C=\mathrm{W})$; GC-MS $(70 \mathrm{eV}) \mathrm{m} / \mathrm{z}(\%) 271\left(\mathrm{M}^{+}-\right.$ $\left.\mathrm{WC}_{5} \mathrm{O}_{5}, 4\right), 228$ (100), 200 (6), 157 (11), 77(21); FT-IR (film, $\mathrm{cm}^{-1}$ ): 2967, 2066, 1931, 1662 (s, $\mathrm{C}=\mathrm{N})$, 752, 699. Anal. calcd for $\mathrm{C}_{22} \mathrm{H}_{21} \mathrm{NO}_{7} \mathrm{~W}$ : C, 44.39; H, 3.56; N, 2.35. Found: C, 44.73; H, $3.62 ; \mathrm{N}, 2.30$

Pentacarbonyl-\{[(1R*,2R*,3S*)-2-(4,5-dihydro-4,4-dimethyl-2-oxazolyl)-3-(4-methoxyphenyl)2-methylcyclopropyl]methoxymethylene\}tungsten (4e): yellow solid, mp 48-49 $\mathrm{C}, 85 \%$. ${ }^{1} \mathrm{H}$ NMR (300 MHz, C $\left.6 \mathrm{D}_{6}\right) \delta 0.92(\mathrm{~s}, 3 \mathrm{H}), 1.09(\mathrm{~s}, 3 \mathrm{H}), 1.48(\mathrm{~s}, 3 \mathrm{H}), 3.22(\mathrm{~d}, J=6.8 \mathrm{~Hz}, 1 \mathrm{H}), 3.33$ and $3.49(2 \times \mathrm{d}, \mathrm{AB}$ system, $J=8.1 \mathrm{~Hz}, 2 \mathrm{H}), 3.36(\mathrm{~s}, 3 \mathrm{H}), 3.92(\mathrm{~s}, 3 \mathrm{H}), 5.21(\mathrm{~d}, J=6.8 \mathrm{~Hz}, 1 \mathrm{H})$, $6.81(\mathrm{~d}, J=8.9 \mathrm{~Hz}, 2 \mathrm{H}), 7.25(\mathrm{~d}, J=8.9 \mathrm{~Hz}, 2 \mathrm{H}) ;{ }^{13} \mathrm{C} \mathrm{NMR}\left(75.4 \mathrm{MHz}, \mathrm{C}_{6} \mathrm{D}_{6}\right) \delta 16.6\left(C \mathrm{H}_{3}\right), 28.7$ $\left(\mathrm{CH}_{3}\right), 28.9\left(\mathrm{CH}_{3}\right), 40.9(\mathrm{CH}), 41.2\left(C_{q}\right), 55.4\left(\mathrm{CH}_{3}\right), 60.0\left(\mathrm{CH}_{3}\right), 68.1(\mathrm{CH}), 69.7\left(C_{q}\right), 80.1\left(\mathrm{CH}_{2}\right)$, $114.4(2 \times C H), 128.8(2 \times C H), 131.0\left(C_{q}\right), 159.9\left(C_{q}\right), 163.8(C=\mathrm{N}), 198.4(4 \times C=\mathrm{O}), 204.8$ 
$(C=\mathrm{O}), 327.1(C=\mathrm{W})$; GC-MS (70 eV) m/z $(\%), 301\left(\mathrm{M}^{+}-\mathrm{WC}_{5} \mathrm{O}_{5}, 6\right), 258$ (100), $230(8), 187(9)$, 159 (9), 127 (8), 115 (5); FT-IR (film, $\mathrm{cm}^{-1}$ ): 2967, 2066, 1931, 1660 (s, C=N), 1230, 1108. Anal. calcd. for $\mathrm{C}_{23} \mathrm{H}_{23} \mathrm{NO}_{8} \mathrm{~W}$ : C, 44.18; H, 3.71; N, 2.24. Found: C, 44.02; H, 3.51; N, 2.02.

\section{Methyl-(1 $\left.R^{*}, 2 R^{*}, 3 S^{*}\right)$-2-(4,5-dihydro-4,4-dimethyl-2-oxazolyl)-2-methyl-3-}

phenylcyclopropanecarboxylate (5a): white solid, mp 53-54 ${ }^{\circ} \mathrm{C}, 72 \% .{ }^{1} \mathrm{H} \mathrm{NMR}(300 \mathrm{MHz}) \delta$ $0.81(\mathrm{~s}, 3 \mathrm{H}), 1.06$ (s, $3 \mathrm{H}), 1.62(\mathrm{~s}, 3 \mathrm{H}), 2.86(\mathrm{~d}, J=6.6 \mathrm{~Hz}, 1 \mathrm{H}), 3.10(\mathrm{~d}, J=6.6 \mathrm{~Hz}, 1 \mathrm{H}), 3.46$ and $3.55(2 \times \mathrm{d}, \mathrm{AB}$ system, $J=7.9 \mathrm{~Hz}, 2 \mathrm{H}), 3.74(\mathrm{~s}, 3 \mathrm{H}), 7.22-7.30(\mathrm{~m}, 5 \mathrm{H}) ;{ }^{13} \mathrm{C}$ NMR $(75.4$ MHz) $\delta 15.6\left(\mathrm{CH}_{3}\right), 28.1\left(\mathrm{CH}_{3}\right), 28.2\left(\mathrm{CH}_{3}\right), 29.6(\mathrm{CH}), 31.1\left(C_{q}\right), 36.9(\mathrm{CH}), 52.0\left(\mathrm{CH}_{3}\right), 66.9\left(C_{q}\right)$, $78.9\left(\mathrm{CH}_{2}\right), 127.3(\mathrm{CH}), 128.1(2 \times C \mathrm{H}), 129.2(2 \times C \mathrm{H}), 135.3\left(C_{q}\right), 163.4(C=\mathrm{N}), 171.8(C=\mathrm{O})$; GC-MS (70 eV) m/z (\%) $287\left(\mathrm{M}^{+}, 5\right), 229$ (28), 228 (100), 200 (13), 157 (13), 129 (20), 115 (8); FT-IR (film, $\mathrm{cm}^{-1}$ ): 2957, 2927, $1732(\mathrm{~s}, \mathrm{C}=\mathrm{O}), 1660(\mathrm{~s}, \mathrm{C}=\mathrm{N}), 1450,1302$. Anal. calcd. for $\mathrm{C}_{17} \mathrm{H}_{21} \mathrm{NO}_{3}: \mathrm{C}, 71.06 ; \mathrm{H}, 7.37 ; \mathrm{N}, 4.87$. Found: $\mathrm{C}, 71.42 ; \mathrm{H}, 7.50 ; \mathrm{N}, 4.80$.

\section{Methyl-(1 $\left.R^{*}, 2 R^{*}, 3 S^{*}\right)-2-(4,5-d i h y d r o-4,4-d i m e t h y l-2-o x a z o l y l)-3-(4-m e t h o x y p h e n y l)-2-$}

methylcyclopropanecarboxylate (5b): yellow solid, 57-59 ${ }^{\circ} \mathrm{C}, 85 \% .{ }^{1} \mathrm{H}$ NMR $(300 \mathrm{MHz}) \delta 0.85$ (s, $3 \mathrm{H}), 1.09(\mathrm{~s}, 3 \mathrm{H}), 1.60(\mathrm{~s}, 3 \mathrm{H}), 2.79(\mathrm{~d}, J=6.6 \mathrm{~Hz}, 1 \mathrm{H}), 3.03(\mathrm{~d}, J=6.6 \mathrm{~Hz}, 1 \mathrm{H}), 3.48$ and $3.58(2 \times \mathrm{d}, \mathrm{AB}$ system, $J=7.8 \mathrm{~Hz}, 2 \mathrm{H}), 3.74(\mathrm{~s}, 3 \mathrm{H}), 3.75(\mathrm{~s}, 3 \mathrm{H}), 6.76(\mathrm{~d}, J=8.9 \mathrm{~Hz}, 2 \mathrm{H}), 7.12$ $(\mathrm{d}, J=8.9 \mathrm{~Hz}, 2 \mathrm{H}) ;{ }^{13} \mathrm{C}$ NMR $(75.4 \mathrm{MHz}) \delta 15.7\left(\mathrm{CH}_{3}\right), 27.9\left(C_{3}\right), 28.2\left(C_{3}\right), 29.3(C \mathrm{H}), 30.8$ $\left(C_{q}\right), 36.5(\mathrm{CH}), 52.0\left(\mathrm{CH}_{3}\right), 55.2\left(\mathrm{CH}_{3}\right), 66.8\left(C_{q}\right), 78.9\left(\mathrm{CH}_{2}\right), 113.2(2 \times C \mathrm{H}), 127.5(2 \times C \mathrm{H})$, $129.7\left(C_{q}\right), 158.4\left(C_{q}\right), 164.0(C=\mathrm{N}), 171.3(C=\mathrm{O})$; GC-MS $(70 \mathrm{eV}) \mathrm{m} / z(\%) 317\left(\mathrm{M}^{+}, 12\right), 286(7)$, 258 (100), 230 (16), 187 (17), 159 (15), 127 (12); FT-IR (film, $\mathrm{cm}^{-1}$ ): 2967, 1732 (s, C=O), 1658 (s, $\mathrm{C}=\mathrm{N}), 1441,1408,1171,810$. Anal. calcd. for $\mathrm{C}_{18} \mathrm{H}_{23} \mathrm{NO}_{4}: \mathrm{C}, 68.12 ; \mathrm{H}, 7.30 ; \mathrm{N}$, 4.41. Found: $\mathrm{C}$, $68.44 ; \mathrm{H}, 7.56 ; \mathrm{N}, 4.22$

Methyl-(1 $\left.R^{*}, 2 R^{*}, 3 S^{*}\right)-(4,5-$ dihydro-4,4-dimethyl-2-oxazolyl)-3-(2-furyl)-2-

methylcyclopropanecarboxylate (5c): colorless oil, $83 \% .{ }^{1} \mathrm{H}$ NMR $(300 \mathrm{MHz}) \delta 0.99$ (s, $\left.3 \mathrm{H}\right)$, 
$1.15(\mathrm{~s}, 3 \mathrm{H}), 1.55(\mathrm{~s}, 3 \mathrm{H}), 2.72(\mathrm{~d}, J=6.4 \mathrm{~Hz}, 1 \mathrm{H}), 3.02(\mathrm{~d}, J=6.4 \mathrm{~Hz}, 1 \mathrm{H}), 3.66$ and $3.71(2 \times \mathrm{d}$, AB system, $J=7.9 \mathrm{~Hz}, 2 \mathrm{H}), 3.73(\mathrm{~s}, 3 \mathrm{H}), 6.04-6.08$ (m, $1 \mathrm{H}), 6.20-6.25$ (m, $1 \mathrm{H}), 7.24-7.30$ (m, $1 \mathrm{H}) ;{ }^{13} \mathrm{C} \mathrm{NMR}(75.4 \mathrm{MHz}) \delta 15.2\left(\mathrm{CH}_{3}\right), 28.0\left(\mathrm{CH}_{3}\right), 28.1\left(\mathrm{CH}_{3}\right), 29.6(\mathrm{CH}), 29.7(\mathrm{CH}), 30.4\left(C_{q}\right)$, $52.0\left(\mathrm{CH}_{3}\right), 66.9\left(C_{q}\right), 79.4\left(\mathrm{CH}_{2}\right), 107.3(\mathrm{CH}), 110.1(C \mathrm{H}), 141.5(C \mathrm{H}), 150.1\left(C_{q}\right), 163.9(C=\mathrm{N})$, $170.5(C=\mathrm{O})$; GC-MS (70 eV) m/z (\%) $277\left(\mathrm{M}^{+}, 5\right), 246$ (3), 218 (100), 190 (11), 147 (10), 129 (20), 91 (9); FT-IR (film, $\mathrm{cm}^{-1}$ ): 2956, 2920, 1732 (s, C=O), 1649 (s, C=N), 1436, 1378, 1205, 919. Anal. calcd. for $\mathrm{C}_{15} \mathrm{H}_{19} \mathrm{NO}_{4}$ : C, 64.97; H, 6.91; N, 5.05. Found: C, 65.29; H, 7.02; N, 4.99.

colorless oil, $49 \%$ inseparable mixture of two diasteroisomers, $\operatorname{dr}\left(1 R^{*}, 2 R^{*}, 3 S^{*}\right) /\left(1 R^{*}, 2 S^{*}, 3 S^{*}\right)$ 1/1. ${ }^{1} \mathrm{H}$ NMR (300 MHz) $\delta 0.86$ (s, $\left.3 \mathrm{H}\right), 1.15$ (s, $\left.3 \mathrm{H}\right), 1.24$ (s, $\left.3 \mathrm{H}\right), 1.27$ (s, $\left.3 \mathrm{H}\right), 2.50-2.61$ (m, 2 H), 2.74-2.85 (m, $2 \mathrm{H}), 2.91-3.06(\mathrm{~m}, 2 \mathrm{H}), 3.50(\mathrm{~s}, 3 \mathrm{H}), 3.52(\mathrm{~s}, 3 \mathrm{H}), 3.58$ and $3.67(2 \times \mathrm{d}, \mathrm{AB}$ system, $J=8.0 \mathrm{~Hz}, 2 \mathrm{H}), 3.99(\mathrm{~s}, 2 \mathrm{H}), 7.02-7.40(\mathrm{~m}, 10 \mathrm{H}) ;{ }^{13} \mathrm{C} \mathrm{NMR}(75.4 \mathrm{MHz}) \delta, 24.6(\mathrm{CH})$, $24.9(\mathrm{CH}), 27.8\left(2 \times \mathrm{CH}_{3}\right), 27.9\left(\mathrm{CH}_{3}\right), 28.0\left(\mathrm{CH}_{3}\right), 29.1(\mathrm{CH}), 29.2(\mathrm{CH}), 31.2(\mathrm{CH}), 31.5(\mathrm{CH})$, $51.8\left(\mathrm{CH}_{3}\right), 52.2\left(\mathrm{CH}_{3}\right), 66.9\left(C_{q}\right), 67.1\left(C_{q}\right), 79.0\left(\mathrm{CH}_{2}\right), 79.2\left(\mathrm{CH}_{2}\right), 127.0(\mathrm{CH}), 127.3(\mathrm{CH})$, $128.1(2 \times C \mathrm{H}), 128.2(2 \times C \mathrm{H}), 129.1(2 \times C \mathrm{H}), 129.2(2 \times C \mathrm{H}), 134.8\left(C_{q}\right), 135.3\left(C_{q}\right), 163.4$ $(C=\mathrm{N}), 163.7(C=\mathrm{N}), 171.2(C=\mathrm{O}), 172.5(C=\mathrm{O})$; GC-MS $(70 \mathrm{eV}) \mathrm{m} / z(\%)$ diastereoisomer with a lower $t_{r}, 273\left(\mathrm{M}^{+}, 8\right), 215(30), 204$ (100),158 (16), 143 (16), 77 (10); diastereoisomer with a higher $t_{r}, 273\left(\mathrm{M}^{+}, 10\right), 242$ (8), 215 (29), 204 (100), 186 (13), 115 (16), 77 (19); FT-IR (film, cm ${ }^{-1}$ ): 2965, 1731 (s, C=O), 1660 (s, C=N), 1652, 1249, 1175, 750, 700. Anal. Calcd for: $\mathrm{C}_{16} \mathrm{H}_{19} \mathrm{NO}_{3}$ : C, 70.31; H, 7.01; N, 5.12. Found: C, 70.53; H, 7.29; N, 5.03.

\section{Methyl-2-(4,5-dihydro-4,4-dimethyl-2-oxazolyl)-3-(4-}

methoxyphenyl)cyclopropanecarboxylate 6b: colorless oil, $53 \%$ inseparable mixture of two diasteroisomers, $\mathrm{dr}\left(1 R^{*}, 2 R^{*}, 3 S^{*}\right) /\left(1 R^{*}, 2 S^{*}, 3 S^{*}\right) 1 / 1 .{ }^{1} \mathrm{H} \mathrm{NMR}(300 \mathrm{MHz}) \delta 0.91(\mathrm{~s}, 3 \mathrm{H}), 1.15(\mathrm{~s}$,

$3 \mathrm{H}), 1.27(\mathrm{~s}, 6 \mathrm{H}), 2.56\left(\mathrm{dd},{ }^{3} J=5.1 \mathrm{~Hz},{ }^{3} J^{\prime}=5.3 \mathrm{~Hz}, 2 \mathrm{H}\right), 2.82\left(\mathrm{dd},{ }^{3} J=5.3 \mathrm{~Hz},{ }^{3} J^{\prime}=5.4 \mathrm{~Hz}, 2\right.$ H), 2.92-3.04 (m, $2 \mathrm{H}), 3.50(\mathrm{~s}, 3 \mathrm{H}), 3.58$ and $3.67(2 \times \mathrm{d}, \mathrm{AB}$ system, $J=8.1 \mathrm{~Hz}, 2 \mathrm{H}), 3.76(\mathrm{~s}, 3$ 
H), $3.79(6 \mathrm{H}), 3.95$ (s, $2 \mathrm{H}), 6.77-6.86(\mathrm{~m}, 4 \mathrm{H}), 7.16-7.24(\mathrm{~m}, 4 \mathrm{H}) ;{ }^{13} \mathrm{C} \mathrm{NMR}(75.4 \mathrm{MHz}) \delta 24.7$ $(C \mathrm{H}), 25.0(\mathrm{CH}), 27.9\left(\mathrm{CH}_{3}\right), 28.1\left(\mathrm{CH}_{3}\right), 28.3\left(\mathrm{CH}_{3}\right), 28.9\left(\mathrm{CH}_{3}\right), 29.3(\mathrm{CH}), 29.5(\mathrm{CH}), 31.1(\mathrm{CH})$, $31.4(\mathrm{CH}), 51.8\left(\mathrm{CH}_{3}\right), 52.2\left(\mathrm{CH}_{3}\right), 55.1\left(\mathrm{CH}_{3}\right), 55.2\left(\mathrm{CH}_{3}\right), 66.9\left(C_{q}\right), 67.1\left(C_{q}\right), 79.0\left(C_{2}\right), 79.2$ $\left(\mathrm{CH}_{2}\right), 113.3(2 \times \mathrm{CH}), 113.5(2 \times \mathrm{CH}), 129.8(2 \times \mathrm{CH}), 129.9(2 \times C \mathrm{H}), 137.3\left(C_{q}\right), 137.4\left(C_{q}\right)$, $158.6\left(2 \times C_{q}\right), 163.5(C=\mathrm{N}), 163.7(C=\mathrm{N}) 172.2(C=\mathrm{O}), 172.5(C=\mathrm{O}) ; \mathrm{GC}-\mathrm{MS}(70 \mathrm{eV}) \mathrm{m} / z(\%)$ diastereoisomer with a lower $t_{r}, 303\left(\mathrm{M}^{+}, 8\right), 272(8), 245$ (30), 244 (100), $216(13), 188(16), 173$ (16), 145 (14), 113 (17); diastereoisomer with a higher $t_{r}, 303\left(\mathrm{M}^{+}, 10\right), 272$ (8), 245 (29), 244 (100), 216 (13), 188 (19), 173 (16), 145 (16), 113 (19); FT-IR (film, cm²): 2965, 1733 (s, C=O), $1663(\mathrm{~s}, \mathrm{C}=\mathrm{N}), 1657(\mathrm{~s}, \mathrm{C}=\mathrm{N})$ 1518, 1249, 1175. Anal. Calcd. for $\mathrm{C}_{17} \mathrm{H}_{21} \mathrm{NO}_{4}: \mathrm{C}, 67.31 ; \mathrm{H}, 6.98 ; \mathrm{N}$, 4.62. Found: C, 67.58; H, 7.21; N, 4.60.

Methyl-(1R,2R,3S,4'S)- and (1S,2S,3R,4'S)-2-[4,5-dihydro-4-isopropyl-2-oxazolyl]-2-methyl-3phenylcyclopopanecarboxylate (7a). Major diastereoisomer: colorless oil, $45 \%$. ${ }^{1} \mathrm{H}$ NMR (300 MHz) $\delta 0.60(\mathrm{~d}, J=6.5 \mathrm{~Hz}, 3 \mathrm{H}), 0.74(\mathrm{~d}, J=6.5 \mathrm{~Hz}, 3 \mathrm{H}), 1.25-1.56(\mathrm{~m}, 1 \mathrm{H}), 1.66$ (s $3 \mathrm{H}), 2.90$ (d, $J=6.5 \mathrm{~Hz}, 1 \mathrm{H}), 3.15(\mathrm{~d}, J=6.5 \mathrm{~Hz}, 1 \mathrm{H}), 3.41-3.92(\mathrm{~m}, 3 \mathrm{H}), 3.80$ (s, $3 \mathrm{H}$ ), 7.20-7.37 (m, 5 $\mathrm{H}) ;{ }^{13} \mathrm{C}$ NMR $(75.4 \mathrm{MHz}) \delta 15.8\left(\mathrm{CH}_{3}\right), 18.1\left(\mathrm{CH}_{3}\right), 18.9\left(\mathrm{CH}_{3}\right), 29.5(C \mathrm{H}), 31.1\left(C_{q}\right), 32.7(C \mathrm{H})$, $37.4(\mathrm{CH}), 52.0\left(\mathrm{CH}_{3}\right), 69.6(\mathrm{CH}), 70.6\left(\mathrm{CH}_{2}\right), 127.8(\mathrm{CH}), 128.4(2 \times \mathrm{CH}), 128.7(2 \times \mathrm{CH}), 135.4$ $\left(C_{q}\right), 163.3(C=\mathrm{N}), 171.2(C=\mathrm{O})$; GC-MS (70 eV) m/z (\%), $301\left(\mathrm{M}^{+}, 11\right), 233(38), 232(100), 204$ (8), 77 (10); FT-IR (film, cm²): 2961, 1729 (s, C=O), 1653 (s, C=N), 1444, 1153, 754, 699. Anal. calcd for $\mathrm{C}_{18} \mathrm{H}_{23} \mathrm{NO}_{3}$ : C, 71.73; H, 7.69; N, 4.65. Found: C, 71.95; H, 7.53; N, 4.37. Minor diastereoisomer: colorless oil, $10 \% .{ }^{1} \mathrm{H}$ NMR $(300 \mathrm{MHz}) \delta 0.67(\mathrm{~d}, J=6.6 \mathrm{~Hz}, 3 \mathrm{H}), 0.82(\mathrm{~d}, J=$ $6.6 \mathrm{~Hz}, 3 \mathrm{H}), 1.21-1.53(\mathrm{~m}, 1 \mathrm{H}), 1.68(\mathrm{~s} 3 \mathrm{H}), 2.90(\mathrm{~d}, J=6.5 \mathrm{~Hz}, 1 \mathrm{H}), 3.09$ (d, J = 6.5 Hz, $1 \mathrm{H})$, 3.38-3.90 (m, $3 \mathrm{H}), 3.82(\mathrm{~s}, 3 \mathrm{H}), 7.23-7.43(\mathrm{~m}, 5 \mathrm{H}) ;{ }^{13} \mathrm{C} \mathrm{NMR}(75.4 \mathrm{MHz}) \delta 15.7\left(\mathrm{CH}_{3}\right), 18.1$ $\left(\mathrm{CH}_{3}\right), 18.9\left(\mathrm{CH}_{3}\right), 29.5(\mathrm{CH}), 31.2\left(\mathrm{C}_{q}\right), 32.7(\mathrm{CH}), 37.4(\mathrm{CH}), 51.9\left(\mathrm{CH}_{3}\right), 69.6(\mathrm{CH}), 72.0\left(\mathrm{CH}_{2}\right)$, $126.8(C H), 128.4(2 \times C H), 128.6(2 \times C H), 132.6\left(C_{q}\right), 163.0(C=\mathrm{N}), 171.1(C=\mathrm{O}) ; \mathrm{GC}-\mathrm{MS}(70$ 
eV) $\mathrm{m} / z(\%), 301\left(\mathrm{M}^{+}, 5\right), 233$ (20), 232 (100), 204 (3), 77 (15); FT-IR (film, cm ${ }^{-1}$ ): 2964, 1727 (s, $\mathrm{C}=\mathrm{O}), 1655(\mathrm{~s}, \mathrm{C}=\mathrm{N}), 1440,1153,750,702$. Anal. calcd for $\mathrm{C}_{18} \mathrm{H}_{23} \mathrm{NO}_{3}: \mathrm{C}, 71.73 ; \mathrm{H}, 7.69 ; \mathrm{N}, 4.65$. Found: C, 72.07; H, 7.74; N, 4.41.

Methyl-(1R,2R,3S,4'S)- $\quad$ and $\quad(1 S, 2 S, 3 R, 4 ' S)-2-[(4,5-d i h y d r o-4-i s o p r o p y l-2-o x a z o l y l]-3-(4-$ methoxyphenyl)-2-methyl-cyclopopanecarboxylate (7b). Major diastereoisomer : white solid, mp $47-48{ }^{\circ} \mathrm{C}, 49 \% .{ }^{1} \mathrm{H}$ NMR $(300 \mathrm{MHz}) \delta 0.62(\mathrm{~d}, 6.5 \mathrm{~Hz}, 3 \mathrm{H}), 0.75(\mathrm{~d}, 6.5 \mathrm{~Hz}, 3 \mathrm{H}), 1.22-1.55$ (m, $1 \mathrm{H}), 1.63(\mathrm{~s}, 3 \mathrm{H}), 2.84(\mathrm{~d}, J=6.5 \mathrm{~Hz}, 1 \mathrm{H}), 3.08(\mathrm{~d}, J=6.5 \mathrm{~Hz}, 1 \mathrm{H}), 3.48-3.92(\mathrm{~m}, 3 \mathrm{H}), 3.78$ (s, $3 \mathrm{H}), 3.80(\mathrm{~s}, 3 \mathrm{H}), 6.70-6.85$ (m, $2 \mathrm{H}), 7.15-7.20(\mathrm{~m}, 2 \mathrm{H}) ;{ }^{13} \mathrm{C}$ NMR $(75.4 \mathrm{MHz}) \delta 16.2\left(\mathrm{CH}_{3}\right)$, $18.7\left(\mathrm{CH}_{3}\right), 18.9\left(\mathrm{CH}_{3}\right), 29.5(\mathrm{CH}), 31.1\left(\mathrm{C}_{q}\right), 32.6(\mathrm{CH}), 36.6(\mathrm{CH}), 51.9\left(\mathrm{CH}_{3}\right), 55.1\left(\mathrm{CH}_{3}\right), 69.4$ $(C \mathrm{H}), 71.9(\mathrm{CH}), 113.3(2 \times C \mathrm{H}), 127.5(2 \times C \mathrm{H}), 129.6\left(C_{q}\right), 158.3\left(C_{q}\right), 165.0(C=\mathrm{N}), 172.2$ $(C=\mathrm{O})$; GC-MS (70 eV) m/z (\%), $331\left(\mathrm{M}^{+}, 11\right), 273$ (38), 272 (100), 244 (3), 159 (12), 127 (8); FTIR (film, $\mathrm{cm}^{-1}$ ): 2957, 1732 (s, C=O), $1661(\mathrm{~s}, \mathrm{C}=\mathrm{N}), 1516,1443,1250,1173,818$. Anal calcd. for $\mathrm{C}_{19} \mathrm{H}_{25} \mathrm{NO}_{4}$ : C, 68.86; H, 7.60; N, 4.23. Found: C, 69.21; H, 7.51; N, 4.10. Minor diastereoisomer: white solid, mp $45-46{ }^{\circ} \mathrm{C}, 11 \% .{ }^{1} \mathrm{H}$ NMR $(300 \mathrm{MHz}) \delta 0.69(\mathrm{~d}, J=6.6 \mathrm{~Hz}, 3 \mathrm{H}), 0.84(\mathrm{~d}, J=6.6$ $\mathrm{Hz}, 3 \mathrm{H}), 1.24-1.56(\mathrm{~m}, 1 \mathrm{H}), 1.65(\mathrm{~s} 3 \mathrm{H}), 2.84(\mathrm{~d}, J=6.5 \mathrm{~Hz}, 1 \mathrm{H}), 3.03(\mathrm{~d}, J=6.5 \mathrm{~Hz}, 1 \mathrm{H})$, 3.46-3.92 (m, $3 \mathrm{H}), 3.79(\mathrm{~s}, 3 \mathrm{H}), 3.81(\mathrm{~s}, 3 \mathrm{H}), 6.68-6.83(\mathrm{~m}, 2 \mathrm{H}), 7.14-7.20(\mathrm{~m}, 2 \mathrm{H}) ;{ }^{13} \mathrm{C} \mathrm{NMR}$ (75.4 MHz) $\delta 16.1\left(\mathrm{CH}_{3}\right), 18.0\left(\mathrm{CH}_{3}\right), 18.8\left(\mathrm{CH}_{3}\right), 29.5(\mathrm{CH}), 32.1\left(C_{q}\right), 32.6(\mathrm{CH}), 36.4(C \mathrm{H}), 51.8$ $\left(\mathrm{CH}_{3}\right), 55.2\left(\mathrm{CH}_{3}\right), 69.8(\mathrm{CH}), 72.3\left(\mathrm{CH}_{2}\right), 113.1(2 \times \mathrm{CH}), 127.5(2 \times C \mathrm{H}), 129.4\left(C_{q}\right), 158.2\left(C_{q}\right)$, $164.9(C=\mathrm{N}), 171.5(C=\mathrm{O})$; GC-MS (70 eV) m/z $(\%), 331\left(\mathrm{M}^{+}, 5\right), 273(24), 272(100), 244(3), 159$ (8), 127 (6); FT-IR (film, cm ${ }^{-1}$ ): 2957, 1732 (s, C=O), 1661 (s, C=N), 1516, 1443, 1250, 1173, 818. Anal calcd. for $\mathrm{C}_{19} \mathrm{H}_{25} \mathrm{NO}_{4}$ : C, 68.86; H, 7.60; N, 4.23. Found: C, 69.16; H, 7.82; N, 4.16.

Methyl-(1R,2R,3S,4S,5S)- and $(1 S, 2 S, 3 R, 4 S, 5 S)$-2-[4,5-dihydro-4-methoxymethyl-5-phenyl-2oxazolyl]-3-(4-methoxyphenyl)-2-methylcyclopopanecarboxylate (7c): white solid, mp 62-64 ${ }^{\circ} \mathrm{C}, 55 \%$, inseparable mixture of two diastereoisomers $(\mathrm{dr}=3 / 2) .{ }^{1} \mathrm{H}$ NMR $(300 \mathrm{MHz}) \delta 1.48(\mathrm{~s}, 3$ $\mathrm{H}$, minor), 1.50 (s, $3 \mathrm{H}$, major), 2.64 (d, $J=6.4 \mathrm{~Hz}, 1 \mathrm{H}$, minor), 2.70 (d, $J=6.4 \mathrm{~Hz}, 1 \mathrm{H}$, major), 
$3.00(\mathrm{~d}, J=6.4 \mathrm{~Hz}, 1 \mathrm{H}$ major) 3.12 (d, $J=6.4 \mathrm{~Hz}, 1 \mathrm{H}$ minor), 3.32 (s, $3 \mathrm{H}$, major), 3.34 (s, $3 \mathrm{H}$, minor), 3.40-3.62 (m, $2 \mathrm{H}$ major and $2 \mathrm{H}$ minor), 3.52 (s, $3 \mathrm{H}$ major and $3 \mathrm{H}$ minor), 3.70-3.95 (m, $1 \mathrm{H}$ major and $1 \mathrm{H}$ minor), 3.76 (s, $3 \mathrm{H}$, major), 3.80 (s, $3 \mathrm{H}$, minor), 4.82 (d, $J=7.7 \mathrm{~Hz}, 1 \mathrm{H}$, major), 4.86 (d, $J=7.7 \mathrm{~Hz}, 1 \mathrm{H}$, minor), 6.74-6.89 (m, 2 H major and $2 \mathrm{H}$ minor), 7.12-7.24 (m, 2 $\mathrm{H}$ major and $2 \mathrm{H}$ minor), 7.21-7.44 (m, $5 \mathrm{H}$ major and $5 \mathrm{H}$ minor); ${ }^{13} \mathrm{C} \mathrm{NMR}(75.4 \mathrm{MHz}) \delta 21.2$ $\left(\mathrm{CH}_{3}\right.$, minor), $21.3\left(\mathrm{CH}_{3}\right.$, major), $29.9(\mathrm{CH}$, major $), 30.0(\mathrm{CH}$, minor $), 32.1(\mathrm{CH}$, major $), 32.3(\mathrm{CH}$, minor), $39.6\left(C_{q}\right.$, major), $39.7\left(C_{q}\right.$, minor $), 51.9\left(\mathrm{CH}_{3}\right.$, minor $), 52.2\left(\mathrm{CH}_{3}\right.$, major $), 55.4\left(\mathrm{CH}_{3}\right.$, major $)$, 55.6 $\left(\mathrm{CH}_{3}\right.$, minor $), 59.2\left(\mathrm{CH}_{3}\right.$, major $), 59.3\left(\mathrm{CH}_{3}\right.$, minor $), 73.6\left(\mathrm{CH}_{2}\right.$, major $), 73.7\left(\mathrm{CH}_{2}\right.$, minor $), 74.2$ ( $C \mathrm{H}$, major), $74.6(\mathrm{CH}$, minor), $84.1(\mathrm{CH}$, minor $), 84.2(\mathrm{CH}$, major $), 114.3(2 \times C \mathrm{H}$, minor $), 114.5$ $(2 \times \mathrm{CH}$, major), $125.3(2 \times \mathrm{CH}$, minor $), 125.6(2 \times \mathrm{CH}$, major $), 128.1(\mathrm{CH}$, major $), 128.2(\mathrm{CH}$, minor $), 128.6(2 \times \mathrm{CH}$, major $), 128.7(2 \times \mathrm{CH}$, minor $), 129.1(2 \times \mathrm{CH}$, minor $), 129.3(2 \times \mathrm{CH}$, major), $131.0\left(C_{q}\right.$, major $), 131.6\left(C_{q}\right.$, minor $), 140.2\left(C_{q}\right.$, major $), 140.3\left(C_{q}\right.$, minor $), 158.8\left(C_{q}\right.$, minor), $159.4\left(C_{q}\right.$, major), $166.2(C=\mathrm{N}$, minor $), 166.5(C=\mathrm{N}$, major), $171.0(C=\mathrm{O}$, minor), 171.3 $\left(C=\mathrm{O}\right.$, major); GC-MS (70 eV) m/z (\%) diastereoisomer minor, $409\left(\mathrm{M}^{+}, 1\right), 380(10), 337(24), 336$ (100), 308 (3), 159 (8), 77 (6); diastereoisomer major, $409\left(\mathrm{M}^{+}, 1\right), 364$ (10), 337 (20), 336 (100), 223 (8), 77 (6); FT-IR (film, $\mathrm{cm}^{-1}$ ): 2967, 1741 (s, C=O), 1661, 1655 (s, C=N), 1443, 1250, 1173, 818, 703. Anal. Calcd. for $\mathrm{C}_{24} \mathrm{H}_{27} \mathrm{NO}_{5}$ : C, 70.40; H, 6.65; N, 3.42. Found: C, 70.12; H, 6.52; N, 3.56. 